\title{
Article \\ Connectedness and Local Connectedness on Infra Soft Topological Spaces
}

\author{
Tareq M. Al-shami ${ }^{1, *(D)}$ and El-Sayed A. Abo-Tabl ${ }^{2,3}$ \\ 1 Department of Mathematics, Sana'a University, Sana'a P.O. Box 1247, Yemen \\ 2 Department of Mathematics, College of Arts and Science, Methnab, Qassim University, \\ Buridah 51931, Saudi Arabia; a.adotabl@qu.edu.sa \\ 3 Department of Mathematics, Faculty of Science, Assiut University, Assiut 71516, Egypt \\ * Correspondence: t.alshami@su.edu.ye
}

Citation: Al-shami, T.M.; Abo-Tabl,

E.-S.A. Connectedness and Local

Connectedness on Infra Soft

Topological Spaces. Mathematics 2021, 9, 1759. https://doi.org/10.3390/ math9151759

Academic Editor: Francisco Gallego Lupiaňez

Received: 29 June 2021

Accepted: 14 July 2021

Published: 26 July 2021

Publisher's Note: MDPI stays neutral with regard to jurisdictional claims in published maps and institutional affiliations.

Copyright: () 2021 by the authors. Licensee MDPI, Basel, Switzerland. This article is an open access article distributed under the terms and conditions of the Creative Commons Attribution (CC BY) license (https:// creativecommons.org/licenses/by/ $4.0 /)$.

\begin{abstract}
This year, we introduced the concept of infra soft topology as a new generalization of soft topology. To complete our analysis of this space, we devote this paper to presenting the concepts of infra soft connected and infra soft locally connected spaces. We provide some descriptions for infra soft connectedness and elucidate that there is no relationship between an infra soft topological space and its parametric infra topological spaces with respect to the property of infra soft connectedness. We discuss the behaviors of infra soft connected and infra soft locally connected spaces under infra soft homeomorphism maps and a finite product of soft spaces. We complete this manuscript by defining a component of a soft point and establishing its main properties. We determine the conditions under which the number of components is finite or countable, and we discuss under what conditions the infra soft connected subsets are components.
\end{abstract}

Keywords: separated soft sets; infra soft connected space; infra soft locally connected space; component; infra soft topology

\section{Introduction}

Nowadays, researchers deal with the complexities of modeling vague/uncertain problems in different fields such as engineering, economics, medical science, computer science and sociology on a daily basis. Since classical methods are not always successful in addressing these types of problems, some novel approaches were proposed such as fuzzy set, rough set and soft set. Soft set, the core idea of this manuscript, was introduced by Molodtsov [1] in 1999. Since then, researchers have applied soft sets to different areas including decision-making problems [2], medical science [3-6] and computer science [7].

Then, Maji with their co-authors [8] put forward the basic concepts of soft set theory. They defined the operators of the intersection, union and difference between two soft sets and a complement of a soft set. To remove the shortcomings observed in Maji et al.'s work, some researchers and scholars reformulated some of their operators and presented some new types. For example, Ali et al. [9] provided new kinds of these operators in a way that enabled keeping some of the properties and results of crisp set theory in soft set theory. Other contributions have been made to define several types of soft equality relations such as those given in $[10,11]$.

In 2011, Çağman et al. [12] and Shabir and Naz [13] hybridized soft sets and A general topology to initiate the concept of soft topological spaces. They followed different methods to define a soft topology. Çağman et al. defined a soft topology over an absolute soft set and different sets of parameters. However, Shabir and Naz formulated a soft topology over fixed sets of the universe and parameters. In this article, we followed the definition of Shabir and Naz. Al-shami and Kočinac [14] discussed the interchangeable properties between general topology and extended soft topology. Alcantud [15] constructed soft 
topologies from the bases of topologies, and Al-Ghour [16] explored the concept of $\omega^{*}$ paracompactness in soft topologies.

In several cases, we note that some of the conditions of a (soft) topology are dispensable because the required properties of topological concepts are kept under some soft topology extensions such as supra soft topology [17], soft bitopology [18] and infra soft topology [19]. In these extensions, we easily construct examples that show the interrelationships among the topological concepts under study. These reasons motivated us to continuously research within the frame of infra soft topology, which is one of the recent interesting developments of soft topology.

Connectedness, local connectedness and components are among the most important concepts in soft topologies. The authors of [20-22] explored these concepts and established their main properties. Our contribution herein is the analysis of the properties of these three concepts within the frame of infra soft topological spaces. We note the validity of many properties of connected and locally connected spaces and components via infra soft topological structures, which means we could study several topological concepts and reveal the interrelationships among them in this frame, instead of classical and soft topologies. Therefore, in this manuscript, we aimed to perform an exhaustive analysis of infra soft topological structures.

We layout this manuscript as follows. In Section 2, we refer to some notions relating to soft set theory and infra soft topology. In Section 3, we introduce the concept of infra soft connected spaces and provide some of its characteristics. In Section 4, we present the concept of infra soft locally connected spaces and establish their basic features. Their components and main properties are explored in Section 5. Finally, we highlight the achievements of this work and possible upcoming works in Section 6.

\section{Preliminaries}

In this section, we outline the necessary concepts and findings that are associated with soft set theory and infra soft topology.

\subsection{Soft Set Theory}

Definition 1 ([1]). Let $\mathcal{W}$ be a set of parameters, $\mathcal{U}$ a universal set and $2^{\mathcal{U}}$ the power set of $\mathcal{U}$. $A$ soft set over $\mathcal{U}$ is an ordered pair $(\theta, \mathcal{W})$, where $\theta: \mathcal{W} \rightarrow 2^{\mathcal{U}}$ is a crisp map. We express a soft set as follows $(\theta, \mathcal{W})=\left\{(w, \theta(w)): w \in \mathcal{W}\right.$ and $\left.\theta(w) \in 2^{\mathcal{U}}\right\}$.

A family of all soft sets over $\mathcal{U}$ under a set of parameters $\mathcal{W}$ is symbolized by $S\left(\mathcal{U}_{\mathcal{W}}\right)$.

Definition 2 ([9]). A soft set $\left(\theta^{c}, \mathcal{W}\right)$ is called a complement of $(\theta, \mathcal{W})$ provided that a map $\theta^{c}: \mathcal{W} \rightarrow 2^{\mathcal{U}}$ is given by $\theta^{c}(w)=\mathcal{U} \backslash \theta(w)$ for each $w \in \mathcal{W}$.

Definition 3 ([8]). If the image of each parameter of $\mathcal{W}$ under a map $\theta: \mathcal{W} \rightarrow 2^{\mathcal{U}}$ is the universal set $\mathcal{U}$, then $(\theta, \mathcal{W})$ is called the absolute soft set over $\mathcal{U}$. Its complement is called the null soft set. The absolute and null soft sets are symbolized by $\widetilde{\mathcal{U}}$ and $\Phi$, respectively.

Definition 4 ([23-25]). If all components of a soft set are equal (resp. finite, countable), then we called it a stable (resp. finite, countable) soft set. Otherwise, it is called unstable (resp. infinite, uncountable).

Definition 5 ([26]). If the image of a one parameter, say $w$, under a map $P: \mathcal{W} \rightarrow 2^{\mathcal{U}}$ is a singleton set, say $\{u\}$, and the image of each parameter $w^{\prime} \in \mathcal{W} \backslash\{w\}$ is the empty set, then a soft set $(P, \mathcal{W})$ is called a soft point over $\mathcal{U}$. It is briefly symbolized by $P_{w}^{u}$.

Definition $6([13,24])$. There are two non-belonging relations between an element $u \in \mathcal{U}$ and $a$ soft set $(\theta, \mathcal{W})$ defined as follows:

(i) $u \notin(\theta, \mathcal{W})$ if $u \notin \theta(w)$ for some $w \in \mathcal{W}$;

(ii) $u \notin(\theta, \mathcal{W})$ if $u \notin \theta(w)$ for all $w \in \mathcal{W}$. 
Definition 7 ([9]). The intersection of two soft sets $(\theta, \mathcal{W})$ and $(\xi, \mathcal{C})$ over $\mathcal{U}$, symbolized by $(\theta, \mathcal{W}) \widetilde{\cap}(\xi, \mathcal{C})$, is a soft set $(\lambda, \mathcal{D})$, where $\mathcal{D}=\mathcal{W} \cap \mathcal{C} \neq \varnothing$, and a map $\lambda: \mathcal{D} \rightarrow 2^{\mathcal{U}}$ is given by $\lambda(w)=\theta(w) \cap \xi(w)$ for each $w \in \mathcal{D}$.

Definition $8([8])$. The union of two soft sets $(\theta, \mathcal{W})$ and $(\xi, \mathcal{C})$ over $\mathcal{U}$, symbolized by $(\theta, \mathcal{W}) \widetilde{U}(\xi, \mathcal{C})$, is a soft set $(\lambda, \mathcal{D})$, where $\mathcal{D}=\mathcal{W} \cup \mathcal{C}$ and a map $\lambda: \mathcal{D} \rightarrow 2^{\mathcal{U}}$ is given as follows:

$$
\lambda(w)=\left\{\begin{array}{cl}
\theta(w) & : w \in \mathcal{W} \backslash \mathcal{C} \\
\xi(w) & : w \in \mathcal{C} \backslash \mathcal{W} \\
\theta(w) \cup \xi(w) & : w \in \mathcal{W} \cap \mathcal{C}
\end{array}\right.
$$

Definition 9 ([27]). A soft set $(\theta, \mathcal{W})$ is a subset of a soft set $(\xi, \mathcal{C})$, symbolized by $(\theta, \mathcal{W}) \widetilde{\subseteq}(\xi, \mathcal{C})$, if $\mathcal{W} \subseteq \mathcal{C}$ and $\theta(w) \subseteq \xi(w)$ for all $w \in \mathcal{W}$. The soft sets $(\theta, \mathcal{W})$ and $(\xi, \mathcal{C})$ are called soft equal if each is a subset of the other.

Definition 10 ([28]). A family of soft sets is said to have the finite (resp. countable) intersection property if the finite (resp. countable) intersection of any members of this family is non-null.

Definition 11 ([26]). The Cartesian product of $(\theta, \mathcal{W})$ and $(\xi, \mathcal{C})$, symbolized by $(\theta \times \xi, \mathcal{W} \times \mathcal{C})$, is defined as $(\theta \times \xi)\left(w, w^{\prime}\right)=\theta(w) \times \xi\left(w^{\prime}\right)$ for each $\left(w, w^{\prime}\right) \in \mathcal{W} \times \mathcal{C}$.

Definition $12([4])$. A soft map $f_{\varphi}$ from $S\left(\mathcal{U}_{\mathcal{W}}\right)$ to $S\left(\mathcal{V}_{\mathcal{C}}\right)$ is a pair of crisp maps $f$ and $\varphi$, where $f: \mathcal{U} \rightarrow \mathcal{V}, \eta: \mathcal{W} \rightarrow \mathcal{C}$. Let $(\theta, \mathcal{M})$ and $(\xi, \mathcal{N})$ be, respectively, subsets of $S\left(\mathcal{U}_{\mathcal{W}}\right)$ and $S\left(\mathcal{V}_{\mathcal{C}}\right)$. Then, the image of $(\theta, \mathcal{M})$ and pre-image of $(\xi, \mathcal{N})$ are given by the following.

(i) $f_{\varphi}(\theta, \mathcal{M})=(f(\theta), \mathcal{C})$ is a soft set in $S\left(\mathbb{V}_{\mathcal{C}}\right)$ such that:

$$
f(\theta)(\omega)=\left\{\begin{array}{ccc}
\widetilde{U}_{w \in \varphi^{-1}}(\omega) \cap \mathcal{M} f(\theta(w)) & : & \varphi^{-1}(\omega) \neq \varnothing \\
\varnothing & : & \varphi^{-1}(\omega)=\varnothing
\end{array}\right.
$$

for each $\omega \in \mathcal{C}$.

(ii) $f_{\varphi}^{-1}(\xi, \mathcal{N})=\left(f^{-1}(\xi), \mathcal{W}\right)$ is a soft set in $S\left(\mathcal{U}_{\mathcal{W}}\right)$ such that:

$$
f^{-1}(\xi)(w)=\left\{\begin{array}{cll}
f^{-1}(\xi(\varphi(w))) & : & \varphi(w) \in \mathcal{N} \\
\varnothing & : & \varphi(w) \notin \mathcal{N}
\end{array}\right.
$$

for each $w \in \mathcal{W}$.

Definition 13 ([4]). A soft map $f_{\varphi}: S\left(\mathcal{U}_{\mathcal{W}}\right) \rightarrow S\left(\mathcal{V}_{\mathcal{C}}\right)$ is said to be injective (resp. surjective, bijective) if both $f$ and $\varphi$ are injective (resp. surjective, bijective).

\subsection{Infra Soft Topological Spaces}

Definition 14 ([19]). A family $\mathrm{Y}$ of soft sets over $\mathcal{U}$ with $\mathcal{W}$ as a parameters set is said to be an infra soft topology on $\mathcal{U}$ if it is closed under finite intersection and $\Phi$ is a member of $\mathrm{Y}$.

The triple $(\mathcal{U}, \mathrm{Y}, \mathcal{W})$ is called an infra soft topological space (briefly, ISTS). We called a member of $Y$ an infra soft open set and called its complement an infra soft closed set. We called $(\mathcal{U}, \mathrm{Y}, \mathcal{W})$ stable if all its infra soft open sets are stable.

Proposition 1 ([19]). Let $(\mathcal{U}, \mathrm{Y}, \mathcal{W})$ be an ISTS. Then, the collection $\mathrm{Y}_{w}=\{\theta(w):(\theta, \mathcal{W}) \in \mathrm{Y}\}$ forms an infra topology on $\mathcal{U}$ for each $w \in \mathcal{W}$.

We called $\mathrm{Y}_{w}$ a parametric infra topology.

\section{Definition 15 ([19]).}

(i) The intersection of all infra soft closed subsets of $(\mathcal{A}, \Omega, \mathcal{B})$ which contains a soft set $(\theta, \mathcal{B})$ is called the infra soft closure points of $(\theta, \mathcal{B})$. It is denoted by $\operatorname{Cl}(\theta, \mathcal{B})$. 
(ii) The union of all infra soft open subsets of $(\mathcal{A}, \Omega, \mathcal{B})$ which are contained in a soft set $(\theta, \mathcal{B})$ is called the infra soft interior points of $(\theta, \mathcal{B})$. It is denoted by $\operatorname{Int}(\theta, \mathcal{B})$.

Definition 16 ([28]). An ISTS $(\mathcal{A}, \Omega, \mathcal{B})$ is said to be infra soft compact (resp. infra soft Lindelöf) provided that every infra soft open cover of $\widetilde{\mathcal{A}}$ has a finite (resp. countable) sub-cover.

Definition 17 ([19]). Let $(\mathcal{U}, Y, \mathcal{W})$ be an ISTS and $(\theta, \mathcal{W})$ be a non-null subset of $\widetilde{\mathcal{U}}$. Then, $\mathrm{Y}_{(\theta, \mathcal{W})}=\{(\theta, \mathcal{W}) \widetilde{\cap}(\lambda, \mathcal{W}):(\lambda, \mathcal{W}) \in \mathrm{Y}\}$ is called an infra soft relative topology on $(\theta, \mathcal{W})$ and $\left((\theta, \mathcal{W}), Y_{(\theta, \mathcal{W})}, \mathcal{W}\right)$ is called a subspace of $(\mathcal{U}, \mathrm{Y}, \mathcal{W})$

A property is called an infra soft hereditary (ISH) property if it passes from an infra soft topological space to every subspace.

Theorem 1 ([19]). Let $\left(\mathcal{Z}, \mathrm{Y}_{\mathcal{Z}}, \mathcal{W}\right)$ be a subspace of $(\mathcal{U}, \mathrm{Y}, \mathcal{W})$. Then, $(\xi, \mathcal{W})$ is an infra soft closed subset of $\left(\mathcal{Z}, \mathrm{Y}_{\mathcal{Z}}, \mathcal{W}\right)$ if there exists an infra soft closed subset $(\theta, \mathcal{W})$ of $(\mathcal{U}, \mathrm{Y}, \mathcal{W})$ such that $(\xi, \mathcal{W})=\widetilde{\mathcal{Z}} \widetilde{\cap}(\theta, \mathcal{W})$.

Definition 18 ([19]). A soft mapping $f_{\varphi}:(\mathcal{U}, Y, \mathcal{W}) \rightarrow(\mathcal{Z}, \theta, Y)$ is said to be infra soft continuous provided that the pre-image of any infra soft open set is an infra soft open set.

A soft mapping $f_{\varphi}:(\mathcal{U}, Y, \mathcal{W}) \rightarrow(\mathcal{Z}, \theta, Y)$ is said to be an infra soft homeomorphism if it is bijective, infra soft continuous and infra soft open (the image of any infra soft open set is an infra soft open set).

A property is called an infra soft topological property (briefly, IST property) if it is preserved by any infra soft homeomorphism.

Proposition 2. Let $\left\{\left(\mathcal{U}_{k}, Y_{k}, \mathcal{W}_{k}\right): k \in K\right\}$ be a family of ISTSs. Then, $\mathbf{Y}=\left\{\prod_{k \in K}\left(\theta_{k}, \mathcal{W}_{k}\right)\right.$ : $\left.\left(\theta_{k}, \mathcal{W}_{k}\right) \in \tau_{k}\right\}$ is an infra soft topology on $\boldsymbol{A}=\prod_{k \in K} \mathcal{U}_{k}$ under a set of parameters $\boldsymbol{B}=\prod_{k \in K} \mathcal{W}_{k}$.

We called $\mathbf{Y}$ given in proposition above a product of infra soft topologies, and $(\mathbf{A}, \mathbf{Y}, \mathbf{B})$ a product of infra soft spaces.

\section{Infra Soft Connected Spaces}

Through this section, we introduce and characterize the concept of infra soft connected spaces. We demonstrate that the family of infra soft connected sets is closed under a union operator provided that their intersection is non-null. Furthermore, we show that the image of an infra soft connected set is preserved by injective infra soft continuous maps, and prove that the finite product of infra soft connected spaces is infra soft connected. With the aid of examples, we elucidate that there is no relationship between infra soft topology and its parametric infra topologies with respect to possessing the property of infra soft connectedness.

Definition 19. We called $(\theta, \mathcal{W})$ and $(\lambda, \mathcal{W})$ two separated soft subsets of an ISTS $(\mathcal{U}, Y, \mathcal{W})$ provided that $(\theta, \mathcal{W}) \widetilde{\cap} C l(\lambda, \mathcal{W})=\Phi$ and $C l(\theta, \mathcal{W}) \widetilde{\cap}(\lambda, \mathcal{W})=\Phi$.

It is obvious that the two separated soft sets are disjoint; the next example clarifies that the converse is incorrect.

Example 1. Let $\mathrm{Y}=\left\{\widetilde{\mathcal{U}},(\theta, \mathcal{W}) \widetilde{\subseteq} \widetilde{\mathcal{U}}: u_{1} \notin(\theta, \mathcal{W})\right\}$ be an infra soft topology on $\mathcal{U}=$ $\left\{u_{1}, u_{2}, u_{3}, u_{4}\right\}$ with $\mathcal{W}=\left\{w_{1}, w_{2}\right\}$ as a set of parameters. Now, the two soft sets $(\theta, \mathcal{W})=$ $\left\{\left(w_{1},\left\{u_{1}\right\}\right),\left(w_{2},\left\{u_{2}\right\}\right)\right\}$ and $(\lambda, \mathcal{W})=\left\{\left(w_{1},\left\{u_{2}\right\}\right),\left(w_{2},\left\{u_{1}\right\}\right)\right\}$ are disjoint. On the other hand, $\left.(\theta, \mathcal{W}) \widetilde{\cap} C l(\lambda, \mathcal{W})=\left\{\left(w_{1},\left\{u_{1}\right\}\right),\left(w_{2}, \varnothing\right\}\right)\right\}$ and $\operatorname{Cl}(\theta, \mathcal{W}) \widetilde{\cap}(\lambda, \mathcal{W})=\left\{\left(w_{1}, \varnothing\right)\right.$, $\left.\left(w_{2},\left\{u_{1}\right\}\right)\right\}$. Hence, they are not separated. 
Definition 20. An ISTS $(\mathcal{U}, Y, \mathcal{W})$ is said to be infra soft disconnected if $\tilde{\mathcal{U}}$ can be expressed as a union of two non-null separated soft sets, say, $(\theta, \mathcal{W})$ and $(\lambda, \mathcal{W})$. Otherwise, $(\mathcal{U}, Y, \mathcal{W})$ is said to be infra soft connected.

We called $(\theta, \mathcal{W})$ and $(\lambda, \mathcal{W})$ disconnection soft sets of $\widetilde{U}$.

An ISTS $(\mathcal{U}, Y, \mathcal{W})$ given in Example 1 is infra soft connected. In the following, we present an example of an infra soft disconnected space.

Example 2. Let $\mathrm{Y}=\left\{\widetilde{\mathcal{U}},(\theta, \mathcal{W}) \widetilde{\simeq} \widetilde{\mathcal{U}}: u_{1} \notin(\theta, \mathcal{W})\right\}$ be an infra soft topology on $\mathcal{U}=$ $\left\{u_{1}, u_{2}, u_{3}, u_{4}\right\}$ with $\mathcal{W}=\left\{w_{1}, w_{2}\right\}$ as a set of parameters. Now, the two soft sets $(\theta, \mathcal{W})=$ $\left\{\left(w_{1},\left\{u_{1}, u_{2}\right\}\right),\left(w_{2},\left\{u_{3}, u_{4}\right\}\right)\right\}$ and $(\lambda, \mathcal{W})=\left\{\left(w_{1},\left\{u_{3}, u_{4}\right\}\right),\left(w_{2},\left\{u_{1}, u_{2}\right\}\right)\right\}$ are separated and their union is $\widetilde{\mathcal{U}}$. Hence, $(\widetilde{\mathcal{U}}, \mathrm{Y}, \mathcal{W})$ is infra soft disconnected.

Proposition 3. The next statements are identical:

(i) $(\mathcal{U}, \mathrm{Y}, \mathcal{W})$ is infra soft connected;

(ii) Two non-null disjoint infra soft closed sets do not exist such that their union is $\widetilde{\mathcal{U}}$;

(iii) Two non-null disjoint infra soft open sets such that their union is $\widetilde{\mathcal{U}}$ do not exist;

(iv) The only infra soft open and infra soft closed subsets are $\widetilde{\mathcal{U}}$ and $\Phi$.

Proof. $(i) \Rightarrow$ (ii): Consider $(\theta, \mathcal{W})$ and $(\lambda, \mathcal{W})$ as infra soft closed sets such that $(\theta, \mathcal{W}) \widetilde{\bigcap}(\lambda, \mathcal{W})=\Phi$ and $(\theta, \mathcal{W}) \widetilde{\cup}(\lambda, \mathcal{W})=\widetilde{\mathcal{U}}$. Then, $\operatorname{cl}(\theta, \mathcal{W})=(\theta, \mathcal{W})$ and $\operatorname{cl}(\lambda, \mathcal{W})=$ $(\lambda, \mathcal{W})$. Therefore, $(\theta, \mathcal{W})$ and $(\lambda, \mathcal{W})$ are separated soft sets. This contradicts $(i)$, which means that $(i i)$ is true.

(ii) $\Rightarrow$ (iii): Consider $(U, \mathcal{W})$ and $(\lambda, \mathcal{W})$ as infra soft open sets such that $(U, \mathcal{W}) \widetilde{\cap}(\lambda, \mathcal{W})=\Phi$ and $(U, \mathcal{W}) \widetilde{U}(\lambda, \mathcal{W})=\widetilde{\mathcal{U}}$. Then, $\left(\theta^{c}, \mathcal{W}\right)$ and $\left(\lambda^{c}, \mathcal{W}\right)$ are infra soft closed subsets. However, we obtain a contradiction with (ii). This means that (iii) is true.

$($ iii $) \Rightarrow(v i)$ : Consider $(\theta, \mathcal{W})$ as a proper non-null infra soft open and infra soft closed set. Then, $(\theta, \mathcal{W}) \widetilde{\bigcap}\left(\theta^{c}, \mathcal{W}\right)=\Phi$ and $(\theta, \mathcal{W}) \widetilde{\cup}\left(\theta^{c}, \mathcal{W}\right)=\widetilde{\mathcal{U}}$. However, we obtain a contradiction with (iii). This means that $(i v)$ is true.

$(i v) \Rightarrow(i)$ : Suppose that there is an infra soft open and infra soft closed set $(\theta, \mathcal{W})$. Then, $\operatorname{Cl}(\theta, \mathcal{W})=(\theta, \mathcal{W})$ and $\operatorname{Cl}\left(\theta^{c}, \mathcal{W}\right)=\left(\theta^{c}, \mathcal{W}\right)$. Therefore, $(\theta, \mathcal{W})$ and $\left(\theta^{c}, \mathcal{W}\right)$ are separated soft sets such that their union is $\widetilde{\mathcal{U}}$. Consequently, $(\mathcal{U}, \mathrm{Y}, \mathcal{W})$ is infra soft disconnected. Hence, we obtain the desired result.

Proposition 4. Every extended infra soft topological space is infra soft disconnected.

Proof. Let $(\mathcal{U}, \mathrm{Y}, \mathcal{W})$ be an extended infra soft topological space. Without loss of generality, consider $\mathcal{W}=\left\{w_{1}, w_{2}\right\}$. Now, the two soft sets $(\theta, \mathcal{W})=\left\{\left(w_{1}, \mathcal{U}\right),\left(w_{2}, \varnothing\right)\right\}$ and $(\lambda, \mathcal{W})=$ $\left\{\left(w_{1}, \varnothing\right),\left(w_{2}, \mathcal{U}\right)\right\}$ are non-null infra soft open such that $(\theta, \mathcal{W}) \widetilde{U}(\lambda, \mathcal{W})=\widetilde{\mathcal{U}}$. According to Proposition $3,(\widetilde{\mathcal{U}}, \mathrm{Y}, \mathcal{W})$ is infra soft disconnected.

Proposition 5. Let $\left(\mathcal{U}_{1}, \mathrm{Y}_{1}, \mathcal{W}_{1}\right)$ and $\left(\mathcal{U}_{2}, \mathrm{Y}_{2}, \mathcal{W}_{2}\right)$ be two ISTSs. Then:

(i) If $\left(\mathcal{U}_{1}, \mathrm{Y}_{1}, \mathcal{W}_{1}\right)$ is infra soft connected such that $\mathrm{Y}_{2} \subseteq \mathrm{Y}_{1}$, then $\left(\mathcal{U}_{2}, \mathrm{Y}_{2}, \mathcal{W}_{2}\right)$ is infra soft connected;

(ii) If $\left(\mathcal{U}_{1}, \mathrm{Y}_{1}, \mathcal{W}_{1}\right)$ is infra soft disconnected such that $\mathrm{Y}_{1} \subseteq \mathrm{Y}_{2}$, then $\left(\mathcal{U}_{2}, \mathrm{Y}_{2}, \mathcal{W}_{2}\right)$ is infra soft disconnected.

Proof. (i): Let $\left(\mathcal{U}_{1}, \mathrm{Y}_{1}, \mathcal{W}_{1}\right)$ be infra soft connected. According to Proposition 3 , the only infra soft clopen subsets of $\widetilde{\mathcal{U}_{1}}$ are $\Phi$ and $\widetilde{\mathcal{U}_{1}}$. Since $Y_{2} \subseteq Y_{1}$, we obtain that $\Phi$ and $\widetilde{\mathcal{U}_{2}}$ are the only infra soft clopen subsets of $\widetilde{\mathcal{U}_{2}}$. Hence, $\left(\mathcal{U}_{2}, \mathrm{Y}_{2}, \mathcal{W}_{2}\right)$ is infra soft connected.

(ii): Let $\left(\mathcal{U}_{1}, \mathrm{Y}_{1}, \mathcal{W}_{1}\right)$ be infra soft disconnected. Suppose that $\left(\mathcal{U}_{2}, \mathrm{Y}_{2}, \mathcal{W}_{2}\right)$ is infra soft 
connected. Then, the only infra soft clopen subsets of $\widetilde{\mathcal{U}_{2}}$ are $\Phi$ and $\widetilde{\mathcal{U}_{2}}$. This implies that $\Phi$ and $\widetilde{\mathcal{U}_{1}}$ are the only infra soft clopen subsets of $\widetilde{\mathcal{U}_{1}}$ (because $Y_{1} \subseteq \mathrm{Y}_{2}$ ). This contradicts our assumption. Hence, $\left(\mathcal{U}_{2}, Y_{2}, \mathcal{W}_{2}\right)$ is infra soft disconnected.

Definition 21. A subset $(\theta, \mathcal{W})$ of $(\mathcal{U}, Y, \mathcal{W})$ is said to be infra soft disconnected if there exist two non-null separated soft sets such that their union is $(\theta, \mathcal{W})$. Otherwise, $(\theta, \mathcal{W})$ is said to be infra soft connected.

Proposition 6. Let $\left((\mathcal{V}, \mathcal{W}), \mathrm{Y}_{(\mathcal{V}, \mathcal{W})}, \mathcal{W}\right)$ be a subspace of $(\mathcal{U}, \mathrm{Y}, \mathcal{W})$. Consider $\mathrm{Cl}_{\mathcal{V}}$ and $\mathrm{Cl}_{\mathcal{U}}$ are infra soft closure operators in $\left((\mathcal{V}, \mathcal{W}), \mathrm{Y}_{(\mathcal{V}, \mathcal{W})}, \mathcal{W}\right)$ and $(\mathcal{U}, \mathrm{Y}, \mathcal{W})$, respectively. Then, $C l_{\mathcal{V}}(\theta, \mathcal{W})=(\mathcal{V}, \mathcal{W}) \widetilde{\cap} C l_{\mathcal{U}}(\theta, \mathcal{W})$ for each $(\theta, \mathcal{W}) \widetilde{\subseteq}(\mathcal{V}, \mathcal{W})$

Proof. $C l_{\mathcal{V}}(\theta, \mathcal{W})=\widetilde{\cap}\left\{(\delta, \mathcal{W}):(\theta, \mathcal{W}) \widetilde{\subseteq}(\delta, \mathcal{W}) ;(\mathcal{V}, \mathcal{W}) \backslash(\delta, \mathcal{W}) \in \mathrm{Y}_{\mathcal{V}}\right\}$

$$
\begin{aligned}
& =\widetilde{\cap}\left\{(\mathcal{V}, \mathcal{W}) \widetilde{\cap}(\lambda, \mathcal{W}):(\theta, \mathcal{W}) \widetilde{\widetilde{C}}(\mathcal{V}, \mathcal{W}) \widetilde{\cap}(\lambda, \mathcal{W}) ;\left(\lambda^{c}, \mathcal{W}\right) \in \mathrm{Y}\right\} \\
& =\widetilde{\cap}\left\{(\mathcal{V}, \mathcal{W}) \widetilde{\cap}(\lambda, \mathcal{W}):(\theta, \mathcal{W}) \widetilde{\widetilde{\subseteq}}(\lambda, \mathcal{W}) ;\left(\lambda^{c}, \mathcal{W}\right) \in \mathrm{Y}\right\} \\
& \left.=(\mathcal{V}, \mathcal{W}) \widetilde{\cap} \tilde{\cap}\left\{(\lambda, \mathcal{W}):(\theta, \mathcal{W}) \widetilde{\widetilde{\subseteq}}(\lambda, \mathcal{W}) ;\left(\lambda^{c}, \mathcal{W}\right) \in \mathrm{Y}\right\}\right] \\
& =(\mathcal{V}, \mathcal{W}) \widetilde{\cap} C l_{\mathcal{U}}(\theta, \mathcal{W}) \quad \square
\end{aligned}
$$

Corollary 1. A soft subset $(\mathcal{V}, \mathcal{W})$ of $(\mathcal{U}, \mathrm{Y}, \mathcal{W})$ is infra soft connected if $\left((\mathcal{V}, \mathcal{W}), \mathrm{Y}_{(\mathcal{V}, \mathcal{W})}, \mathcal{W}\right)$ is infra soft connected.

Proof. Let $(\delta, \mathcal{W})$ and $(\lambda, \mathcal{W})$ be disconnection soft sets of $(\mathcal{V}, \mathcal{W})$. Then, the proof comes from the fact that:

$$
\begin{gathered}
{\left[(\delta, \mathcal{W}) \widetilde{\cap} C l_{\mathcal{U}}(\lambda, \mathcal{W})\right] \widetilde{\cup}\left[(\lambda, \mathcal{W}) \widetilde{\cap} C l_{\mathcal{U}}(\delta, \mathcal{W})\right]=\Phi \text { if and only if }} \\
{\left[(\delta, \mathcal{W}) \widetilde{\cap} C l_{\mathcal{V}}(\lambda, \mathcal{W})\right] \widetilde{U}\left[(\lambda, \mathcal{W}) \widetilde{\cap} C l_{\mathcal{V}}(\delta, \mathcal{W})\right]=\Phi .}
\end{gathered}
$$

Theorem 2. If $(\theta, \mathcal{W})$ is an infra soft connected set such that $(\theta, \mathcal{W}) \widetilde{\subseteq}(\lambda, \mathcal{W}) \widetilde{\subseteq} C l(\theta, \mathcal{W})$, then $(\lambda, \mathcal{W})$ is infra soft connected.

Proof. Let the given conditions be satisfied. Suppose that $(\lambda, \mathcal{W})$ is infra soft disconnected. Then, there are disconnection soft sets $(\beta, \mathcal{W})$ and $(\delta, \mathcal{W})$ of $(\lambda, \mathcal{W})$. That is, $(\beta, \mathcal{W}) \widetilde{\cup}(\delta, \mathcal{W})=(\lambda, \mathcal{W})$. Since $(\theta, \mathcal{W}) \widetilde{\subseteq}(\lambda, \mathcal{W}),(\beta, \mathcal{W}) \widetilde{\cap}(\theta, \mathcal{W})$ and $(\delta, \mathcal{W}) \widetilde{\cap}(\theta, \mathcal{W})$ are disjoint such that $(\theta, \mathcal{W})=[(\beta, \mathcal{W}) \widetilde{\cap}(\theta, \mathcal{W})] \widetilde{\cup}[(\delta, \mathcal{W}) \widetilde{\cap}(\theta, \mathcal{W})]$. It follows from the infra soft connectedness of $(\theta, \mathcal{W})$ that $(\beta, \mathcal{W}) \widetilde{\cap}(\theta, \mathcal{W})=\Phi$ or $(\delta, \mathcal{W}) \widetilde{\cap}(\theta, \mathcal{W})=\Phi$. For example, $(\beta, \mathcal{W}) \widetilde{\cap}(\theta, \mathcal{W})=\Phi$. This means that $(\theta, \mathcal{W}) \widetilde{\subseteq}(\delta, \mathcal{W})$; consequently, $(\theta, \mathcal{W}) \widetilde{\subseteq}\left(\beta^{c}, \mathcal{W}\right)$. Therefore, $(\theta, \mathcal{W}) \widetilde{\subseteq}(\lambda, \mathcal{W}) \widetilde{\subseteq} C l(\theta, \mathcal{W}) \widetilde{\subseteq}\left(\beta^{c}, \mathcal{W}\right)$. Thus, $(\lambda, \mathcal{W}) \widetilde{\cap}(\beta, \mathcal{W})=\Phi$; this is a contradiction. Hence, $(\lambda, \mathcal{W})$ is infra soft connected.

Definition 22. The difference between infra soft closure points and infra soft interior points of a subset $(\theta, \mathcal{W})$ of $(\mathcal{U}, Y, \mathcal{W})$ is called infra soft boundary points. It is denoted by $\operatorname{Ib}(\theta, \mathcal{W})$.

Proposition 7. If $(\theta, \mathcal{W})$ is a non-null proper subset of an infra soft connected space $(\mathcal{U}, \mathrm{Y}, \mathcal{W})$, then $\operatorname{Ib}(\theta, \mathcal{W}) \neq \Phi$.

Proof. Suppose that $\operatorname{Ib}(\theta, \mathcal{W})=\Phi$. Then, $\operatorname{Cl}(\theta, \mathcal{W})=\operatorname{Int}(\theta, \mathcal{W})$. By hypothesis $\widetilde{\mathcal{U}} \neq$ $(\theta, \mathcal{W}) \neq \Phi$, then $(\theta, \mathcal{W})$ is an infra soft open and infra soft closed set. This is a contradiction with the infra soft connectedness of $(\mathcal{U}, \mathrm{Y}, \mathcal{W})$. Hence, $\operatorname{Ib}(\theta, \mathcal{W}) \neq \Phi$, as required.

Lemma 1. Consider $(\theta, \mathcal{W})$ and $(\lambda, \mathcal{W})$ as disconnection soft sets of $(\mathcal{U}, \mathrm{Y}, \mathcal{W})$. If $(\delta, \mathcal{W})$ is an infra soft connected subset of $(\mathcal{U}, Y, \mathcal{W})$, then $(\theta, \mathcal{W}) \widetilde{\simeq}(\delta, \mathcal{W})$ or $(\lambda, \mathcal{W}) \widetilde{\subseteq}(\delta, \mathcal{W})$.

Proof. Since $(\theta, \mathcal{W})$ and $(\lambda, \mathcal{W})$ are disconnection soft sets of $(\mathcal{U}, Y, \mathcal{W}),(\theta, \mathcal{W}) \widetilde{\cup}(\lambda, \mathcal{W})=$ $\widetilde{\mathcal{U}}$ and $[(\theta, \mathcal{W}) \widetilde{\cap} C l(\lambda, \mathcal{W})] \widetilde{\cup}[C l(\theta, \mathcal{W}) \widetilde{\cap}(\lambda, \mathcal{W})]=\Phi$. So: 


$$
(\delta, \mathcal{W})=[(\delta, \mathcal{W}) \widetilde{\cap}(\theta, \mathcal{W})] \widetilde{\cup}[(\delta, \mathcal{W}) \widetilde{\cap}(\lambda, \mathcal{W})]
$$

Note that $[((\delta, \mathcal{W}) \widetilde{\cap}(\theta, \mathcal{W})) \widetilde{\cap} c l((\delta, \mathcal{W}) \widetilde{\cap}(\theta, \mathcal{W}))] \widetilde{\cup}[((\delta, \mathcal{W}) \widetilde{\cap}(\lambda, \mathcal{W})) \widetilde{\cap} C l((\delta, \mathcal{W}) \widetilde{\cap}(\lambda, \mathcal{W}))]$ $\widetilde{\subseteq}[(\delta, \mathcal{W}) \widetilde{\cap} c l(\theta, \mathcal{W})] \widetilde{\cup}[(\delta, \mathcal{W}) \widetilde{\cap} c l(\lambda, \mathcal{W})]=\Phi$. Since $(\delta, \mathcal{W})$ is infra soft connected, we obtain either $(\delta, \mathcal{W}) \widetilde{\cap}(\theta, \mathcal{W})=\Phi$ or $(\delta, \mathcal{W}) \widetilde{\cap}(\lambda, \mathcal{W})=\Phi$. This automatically implies that $(\delta, \mathcal{W}) \widetilde{\subseteq}(\theta, \mathcal{W})$ or $(\delta, \mathcal{W}) \widetilde{\subseteq}(\lambda, \mathcal{W})$, as required

Theorem 3. If $(\theta, \mathcal{W})$ is a subset of $(\mathcal{U}, Y, \mathcal{W})$ such that for each $P_{w}^{u}, P_{w^{\prime}}^{u^{\prime}} \in(\theta, \mathcal{W})$, there is an infra soft connected subset $(\beta, \mathcal{W})$ of $(\theta, \mathcal{W})$ containing $P_{w}^{u}, P_{w^{\prime}}^{u^{\prime}}$, then $(\theta, \mathcal{W})$ is infra soft connected.

Proof. Let the given conditions be satisfied. Suppose that $(\theta, \mathcal{W})$ is infra soft disconnected. Then, there are disconnection soft sets $(\delta, \mathcal{W})$ and $(\lambda, \mathcal{W})$ of $(\theta, \mathcal{W})$. Therefore, there are two soft points $P_{w}^{u}, P_{w^{\prime}}^{u^{\prime}}$ such that $P_{w}^{u} \in(\delta, \mathcal{W})$ and $P_{w^{\prime}}^{u^{\prime}} \in(\lambda, \mathcal{W})$. By hypothesis, there is an infra soft connected set $(\beta, \mathcal{W})$ containing $P_{w}^{u}, P_{w^{\prime}}^{u^{\prime}}$ such that $(\beta, \mathcal{W}) \widetilde{\subseteq}(\theta, \mathcal{W})=(\delta, \mathcal{W}) \widetilde{\cup}(\lambda, \mathcal{W})$. It comes from the above lemma that $(\beta, \mathcal{W}) \widetilde{\subseteq}(\delta, \mathcal{W})$ or $(\beta, \mathcal{W}) \widetilde{\subseteq}(\lambda, \mathcal{W})$. This leads to $(\delta, \mathcal{W}) \widetilde{\cap}(\lambda, \mathcal{W}) \neq \Phi$. This is a contradiction because $(\delta, \mathcal{W})$ and $(\lambda, \mathcal{W})$ are disconnection soft sets of $(\theta, \mathcal{W})$. Hence, $(\theta, \mathcal{W})$ is infra soft connected.

Corollary 2. If $(\theta, \mathcal{W})$ is a union of infra soft connected sets $\left(\beta_{i}, \mathcal{W}\right)$ such that $\widetilde{\cap}\left(\beta_{i}, \mathcal{W}\right) \neq \Phi$, then $(\theta, \mathcal{W})$ is infra soft connected.

Proof. Let the given conditions be satisfied. Suppose that $(\theta, \mathcal{W})$ is infra soft disconnected. Then, there are disconnection soft sets $(\delta, \mathcal{W})$ and $(\lambda, \mathcal{W})$ of $(\theta, \mathcal{W})$. By hypothesis $\widetilde{\cap}\left(\beta_{i}, \mathcal{W}\right) \neq \Phi$, there exists a soft point $P_{w}^{u}$ such that $P_{w}^{u} \in\left(\beta_{i}, \mathcal{W}\right)$ for each $i$. Now, either $P_{w}^{u} \in(\delta, \mathcal{W})$ or $P_{w}^{u} \in(\lambda, \mathcal{W})$. Say, $P_{w}^{u} \in(\delta, \mathcal{W})$. Then, $\left[\widetilde{\cap}\left(\beta_{i}, \mathcal{W}\right)\right] \widetilde{\cap}(\theta, \mathcal{W}) \neq \Phi$. It comes from Theorem 3 that $\left(\beta_{i}, \mathcal{W}\right) \widetilde{\subseteq}(\delta, \mathcal{W})$ for each $i$. This means that $(\theta, \mathcal{W}) \widetilde{\subseteq}(\delta, \mathcal{W})$. This is a contradiction. Hence, $(\theta, \mathcal{W})$ is infra soft connected.

Proposition 8. The injective infra soft continuous image of an infra soft connected set is infra soft connected.

Proof. Consider $f_{\varphi}:\left(\mathcal{U}_{1}, \mathrm{Y}_{1}, \mathcal{W}_{1}\right) \rightarrow\left(\mathcal{U}_{2}, \mathrm{Y}_{2}, \mathcal{W}_{2}\right)$ as an infra soft continuous map and let $\left(\theta, \mathcal{W}_{1}\right)$ be an infra soft connected subset of $\left(\mathcal{U}_{1}, Y_{1}, \mathcal{W}_{1}\right)$. Suppose that $f_{\varphi}\left(\theta, \mathcal{W}_{1}\right)$ is infra soft disconnected. Then, there are disjoint infra soft open sets $\left(\delta, \mathcal{W}_{2}\right)$ and $\left(\lambda, \mathcal{W}_{2}\right)$ such that $f_{\varphi}\left(\theta, \mathcal{W}_{1}\right)=\left(\delta, \mathcal{W}_{2}\right) \widetilde{\cup}\left(\lambda, \mathcal{W}_{2}\right)$. Now, $f_{\varphi}^{-1}\left(\delta, \mathcal{W}_{2}\right)$ and $f_{\varphi}^{-1}\left(\lambda, \mathcal{W}_{2}\right)$ are two disjoint infra soft open subsets of $\left(\mathcal{U}_{2}, \mathrm{Y}_{2}, \mathcal{W}_{2}\right)$. Since $f_{\varphi}$ is injective, $\left(\theta, \mathcal{W}_{1}\right)=f_{\varphi}^{-1}\left(f_{\varphi}\left(\theta, \mathcal{W}_{1}\right)\right)=$ $f_{\varphi}^{-1}\left(\delta, \mathcal{W}_{2}\right) \widetilde{\cup} f_{\varphi}^{-1}\left(\lambda, \mathcal{W}_{2}\right)$. This means that $\left(\theta, \mathcal{W}_{1}\right)$ is infra soft disconnected; a contradiction. Hence, $f_{\varphi}\left(\theta, \mathcal{W}_{1}\right)$ is infra soft connected.

Corollary 3. The property of being an infra soft connected space is an IST property.

Theorem 4. The finite product of infra soft connected spaces is infra soft connected.

Proof. Let $\left(\mathcal{U}_{1}, \mathrm{Y}_{1}, \mathcal{W}_{1}\right)$ and $\left(\mathcal{U}_{2}, \mathrm{Y}_{2}, \mathcal{W}_{2}\right)$ be two infra soft connected spaces. Suppose that $\mathcal{U}_{1} \times \mathcal{U}_{2}$ is infra soft disconnected. Then, there exist infra soft open subsets $\left(\delta, \mathcal{W}_{1}\right) \times\left(\lambda, \mathcal{W}_{2}\right)$ and $\left(\beta, \mathcal{W}_{1}\right) \times\left(\xi, \mathcal{W}_{2}\right)$ of $\mathcal{U}_{1} \times \mathcal{U}_{2}$ such that $\mathcal{U}_{1} \times \mathcal{U}_{2}=\left[\left(\delta, \mathcal{W}_{1}\right) \times\left(\lambda, \mathcal{W}_{2}\right)\right] \widetilde{U}\left[\left(\beta, \mathcal{W}_{1}\right) \times\right.$ $\left.\left(\xi, \mathcal{W}_{2}\right)\right]$ and $\left[\left(\delta, \mathcal{W}_{1}\right) \times\left(\lambda, \mathcal{W}_{2}\right)\right] \widetilde{\cap}\left[\left(\beta, \mathcal{W}_{1}\right) \times\left(\xi, \mathcal{W}_{2}\right)\right]=\Phi$. Now, $\widetilde{\mathcal{U}}_{1}=\left(\delta, \mathcal{W}_{1}\right) \widetilde{U}\left(\beta, \mathcal{W}_{1}\right)$, $\widetilde{\mathcal{U}}_{2}=\left(\lambda, \mathcal{W}_{2}\right) \widetilde{\cup}\left(\xi, \mathcal{W}_{2}\right)$ and $\left(\delta, \mathcal{W}_{1}\right) \tilde{\cap}\left(\beta, \mathcal{W}_{1}\right)=\Phi$ or $\left(\lambda, \mathcal{W}_{2}\right) \widetilde{\cap}\left(\xi, \mathcal{W}_{2}\right)=\Phi$. This implies that $\left(\mathcal{U}_{1}, \mathrm{Y}_{1}, \mathcal{W}_{1}\right)$ is infra soft disconnected or $\left(\mathcal{U}_{2}, \mathrm{Y}_{2}, \mathcal{W}_{2}\right)$ is infra soft disconnected. This contradicts the assumption. Hence, $\mathcal{U}_{1} \times \mathcal{U}_{2}$ is infra soft connected.

The next examples illustrate that the property of infra soft connected space does not transmit from an ISTS to classical infra topological spaces and vice versa. 
Example 3. The family $\mathrm{Y}=\left\{\Phi, \widetilde{\mathcal{U}},\left(\theta_{k}, \mathcal{W}\right): k=1,2,3,4\right\}$ is an infra soft topology on $\mathcal{U}=$ $\left\{u_{1}, u_{2}, u_{3}\right\}$ with a set of parameters $\mathcal{W}=\left\{w_{1}, w_{2}\right\}$, where:

$$
\begin{aligned}
& \left(\theta_{1}, \mathcal{W}\right)=\left\{\left(w_{1}, \mathcal{U}\right),\left(w_{2}, \varnothing\right)\right\} ; \\
& \left(\theta_{2}, \mathcal{W}\right)=\left\{\left(w_{1}, \varnothing\right),\left(w_{2}, \mathcal{U}\right)\right\} \\
& \left(\theta_{3}, \mathcal{W}\right)=\left\{\left(w_{1},\left\{u_{1}\right\}\right),\left(w_{2}, \varnothing\right)\right\} \text { and } \\
& \left(\theta_{4}, \mathcal{W}\right)=\left\{\left(w_{1}, \varnothing\right),\left(w_{2},\left\{u_{2}\right\}\right)\right\} .
\end{aligned}
$$

Obviously, $(\mathcal{U}, Y, \mathcal{W})$ is an infra soft disconnected. On the other hand, $\left(\mathcal{U}, \mathrm{Y}_{w_{1}}\right)$ and $\left(\mathcal{U}, \mathrm{Y}_{w_{2}}\right)$ are infra connected.

Example 4. The family $\mathrm{Y}=\left\{\Phi, \widetilde{\mathcal{U}},\left(\theta_{k}, \mathcal{W}\right): k=1,2,3,4\right\}$ is an infra soft topology on $\mathcal{U}=$ $\left\{u_{1}, u_{2}, u_{3}\right\}$ with a set of parameters $\mathcal{W}=\left\{w_{1}, w_{2}\right\}$, where:

$$
\begin{aligned}
& \left(\theta_{1}, \mathcal{W}\right)=\left\{\left(w_{1},\left\{u_{1}\right\}\right),\left(w_{2},\left\{u_{2}, u_{3}\right\}\right)\right\} ; \\
& \left(\theta_{2}, \mathcal{W}\right)=\left\{\left(w_{1},\left\{u_{2}, u_{3}\right\}\right),\left(w_{2},\left\{u_{2}\right\}\right)\right\} ; \\
& \left(\theta_{3}, \mathcal{W}\right)=\left\{\left(w_{1}, \varnothing\right),\left(w_{2},\left\{u_{2}\right\}\right)\right\} \text { and } \\
& \left(\theta_{4}, \mathcal{W}\right)=\left\{\left(w_{1}, \varnothing\right),\left(w_{2},\left\{u_{1}\right\}\right)\right\} .
\end{aligned}
$$

Obviously, $(\mathcal{U}, Y, \mathcal{W})$ is infra soft connected. On the other hand, $\left(\mathcal{U}, \mathrm{Y}_{w_{1}}\right)$ and $\left(\mathcal{U}, \mathrm{Y}_{w_{2}}\right)$ are infra disconnected.

Proposition 9. A stable ISTS $(\mathcal{U}, \mathrm{Y}, \mathcal{W})$ is infra soft connected if $\left(\mathcal{U}, \mathrm{Y}_{w}\right)$ is infra connected.

Proof. Straightforward.

Remark 1. Note that the property of being an infra soft connected space is not preserved by a subspace; i.e., it fails to be an ISH property. To validate this matter, take a subset $(\theta, \mathcal{W})=$ $\left\{\left(w_{1},\left\{u_{1}\right\}\right),\left(w_{2},\left\{u_{1}\right\}\right)\right\}$ of an ISTS $(\mathcal{U}, Y, \mathcal{W})$ given in Example 4. Then, $\mathrm{Y}_{(\theta, \mathcal{W})}=\{\Phi,(\theta, \mathcal{W})$, $\left.\left\{\left(w_{1},\left\{u_{1}\right\}\right),\left(w_{2}, \varnothing\right)\right\},\left\{\left(w_{1}, \varnothing\right),\left(w_{2},\left\{u_{1}\right\}\right)\right\}\right\}$. It is clear that $\left\{\left(w_{1},\left\{u_{1}\right\}\right),\left(w_{2}, \varnothing\right)\right\}$ and $\left\{\left(w_{1}, \varnothing\right),\left(w_{2},\left\{u_{1}\right\}\right)\right\}$ are disconnection infra soft sets of a subspace $\left((\theta, \mathcal{W}), Y_{(\theta, \mathcal{W})}, \mathcal{W}\right)$. Hence, $\left((\theta, \mathcal{W}), \mathrm{Y}_{(\theta, \mathcal{W})}, \mathcal{W}\right)$ is infra soft disconnected in spite of $(\mathcal{U}, \mathrm{Y}, \mathcal{W})$ being infra soft connected.

\section{Infra Soft Locally Connected Spaces}

This section is allocated to present the concept of infra soft locally connected spaces and explore their main properties. We characterize it and show that the concepts of infra soft connected and infra soft locally connected spaces are independent of each other. Furthermore, we prove that the property of being an infra soft locally connected space is an infra soft open hereditary and IST property. Finally, we show that the finite product of infra soft locally connected spaces is also infra soft locally connected.

Definition 23. The two soft points are called connected in an ISTS $(\mathcal{U}, Y, \mathcal{W})$ if there exists an infra soft connected subset containing them.

Proposition 10. If every two soft points in an ISTS $(\mathcal{U}, Y, \mathcal{W})$ are connected, then $(\mathcal{U}, Y, \mathcal{W})$ is infra soft connected.

Proof. Let $P_{w}^{u}$ be a fixed soft point in an ISTS $(\mathcal{U}, \mathrm{Y}, \mathcal{W})$. Then, for each soft point $P_{x}^{v}$ different than $P_{w}^{u}\left(P_{w}^{u} \neq P_{x}^{v}\right.$ if $u \neq v$ or $\left.w \neq x\right)$, we have an infra soft connected set $\left(\delta_{v x}, \mathcal{W}\right)$ containing $P_{w}^{u}$ and $P_{x}^{v}$. Since $P_{w}^{u} \in \widetilde{\cap}_{P_{x}^{v} \in \widetilde{\mathcal{U}}}\left(\delta_{v x}, \mathcal{W}\right)$, it follows from Corollary 2 that $\widetilde{\cup}_{P_{x}^{v} \in \widetilde{\mathcal{U}}}\left(\delta_{v x}, \mathcal{W}\right)=\widetilde{\mathcal{U}}$ is infra soft connected.

Definition 24. An ISTS $(\mathcal{U}, Y, \mathcal{W})$ is said to be:

(i) Infra soft locally connected at $P_{w}^{u} \in \widetilde{\mathcal{U}}$ if for every infra soft neighborhood $(\delta, \mathcal{W})$ of $P_{w}^{u}$ there exists an infra soft neighborhood $(\lambda, \mathcal{W}) \widetilde{\subseteq}(\delta, \mathcal{W})$ of $P_{w}^{u}$ such that every two soft points in $(\lambda, \mathcal{W})$ are connected in $(\delta, \mathcal{W})$.

(ii) Infra soft locally connected if it is infra soft locally connected at every soft point $P_{w}^{u}$ in $\tilde{\mathcal{U}}$. Otherwise, it is said to be infra soft locally disconnected. 
The concepts of infra soft connectedness and infra soft locally connectedness are independent from one another. The next two examples explain this fact.

Example 5. An ISTS $(\mathcal{U}, Y, \mathcal{W})$ given in Example 3 is an infra soft locally connected space, but not infra soft connected.

Example 6. The topologist's sine curve is an example of an infra soft connected space which is not infra soft locally connected.

We recall that we called $(\theta, \mathcal{W})$ an infra soft connected neighborhood of a soft point $P_{w}^{u}$ if it is infra soft connected and infra soft neighborhood of $P_{w}^{u}$; i.e., there exists an infra soft open set $(\lambda, \mathcal{W})$ such that $P_{w}^{u} \in(\lambda, \mathcal{W}) \widetilde{\subseteq}(\theta, \mathcal{W})$.

Theorem 5. An ISTS $(\mathcal{U}, Y, \mathcal{W})$ is infra soft locally connected at $P_{w}^{u} \in \widetilde{\mathcal{U}}$ if every infra soft neighborhood of $P_{w}^{u}$ contains an infra soft connected neighborhood of it.

Proof. Necessity: Let $(\mathcal{U}, \mathrm{Y}, \mathcal{W})$ be infra soft locally connected at $P_{w}^{u} \in \tilde{\mathcal{U}}$. Consider $(\theta, \mathcal{W})$ as an infra soft neighborhood of $P_{w}^{u}$. Then, there exists an infra soft neighborhood $(\lambda, \mathcal{W}) \widetilde{\subseteq}(\theta, \mathcal{W})$ of $P_{w}^{u}$ such that every two soft points in $(\lambda, \mathcal{W})$ are connected in $(\theta, \mathcal{W})$. For each soft point $P_{e}^{v} \neq P_{w}^{u}$ in $(\lambda, \mathcal{W})$, there exists an infra soft connected set $\left(\beta_{v x}, \mathcal{W}\right) \widetilde{\subseteq}(\theta, \mathcal{W})$ containing $P_{e}^{v}$ and $P_{w}^{u}$. Putting $(\xi, \mathcal{W})=\widetilde{U}_{P_{e}^{v} \in(\lambda, \mathcal{W})}\left(\beta_{v x}, \mathcal{W}\right)$. Now, $(\lambda, \mathcal{W}) \widetilde{\subseteq}(\xi, \mathcal{W}) \widetilde{\subseteq}(\theta, \mathcal{W})$; according to Corollary $2,(\xi, \mathcal{W})$ is an infra soft connected neighborhood of $P_{w}^{u}$. Thus, we obtain the desired result.

Sufficiency: It comes from Definition 24.

We recall that an ISH property is called an infra soft open hereditary property if it passes from an ISTS to every infra soft open subspace.

Theorem 6. The property of being an infra soft locally connected space is an infra soft open hereditary property.

Proof. Let $\left((\theta, \mathcal{W}), \mathrm{Y}_{(\theta, \mathcal{W})}, \mathcal{W}\right)$ be an infra soft open subspace of an infra soft locally connected space $(\mathcal{U}, Y, \mathcal{W})$. Let $(\lambda, \mathcal{W})$ be an infra soft neighborhood of $P_{w}^{u}$ in $\left((\theta, \mathcal{W}), Y_{(\theta, \mathcal{W})}, \mathcal{W}\right)$. Then, there exists an infra soft open subset $(\delta, \mathcal{W})$ of $(\mathcal{U}, Y, \mathcal{W})$ such that $(\theta, \mathcal{W}) \widetilde{\cap}(\delta, \mathcal{W}) \widetilde{\subseteq}(\lambda, \mathcal{W})$. Since $(\theta, \mathcal{W})$ is an infra soft open set, $(\theta, \mathcal{W}) \widetilde{\cap}(\delta, \mathcal{W})$ is an infra soft open set in $(\mathcal{U}, \mathrm{Y}, \mathcal{W})$; consequently, $(\lambda, \mathcal{W})$ is an infra soft neighborhood of $P_{w}^{u}$ in $(\mathcal{U}, \mathrm{Y}, \mathcal{W})$. By hypothesis of the infra soft local connectedness of $(\mathcal{U}, Y, \mathcal{W})$ there exists an infra soft neighborhood $(\beta, \mathcal{W})$ of $P_{w}^{u}$ in $(\mathcal{U}, Y, \mathcal{W})$ such that every two soft points in $(\beta, \mathcal{W})$ are connected in $(\lambda, \mathcal{W})$. Now, $(\beta, \mathcal{W}) \widetilde{\cap}(\lambda, \mathcal{W})$ is an infra soft neighborhood of $P_{w}^{u}$ in $\left((\theta, \mathcal{W}), \mathrm{Y}_{(\theta, \mathcal{W})}, \mathcal{W}\right)$ such that every two soft points of it are connected in $(\lambda, \mathcal{W})$. Hence, the proof is complete.

Proposition 11. The property of being an infra soft locally connected space is an IST property.

Proof. Consider $f_{\varphi}:\left(\mathcal{U}_{1}, \mathrm{Y}_{1}, \mathcal{W}_{1}\right) \rightarrow\left(\mathcal{U}_{2}, \mathrm{Y}_{2}, \mathcal{W}_{2}\right)$ as an infra soft homomorphism map such that $\left(\mathcal{U}_{1}, Y_{1}, \mathcal{W}_{1}\right)$ is an infra soft locally connected space. Let $\left(\delta, \mathcal{W}_{2}\right)$ be an infra soft neighborhood of $P_{x}^{v} \in \widetilde{\mathcal{U}}_{2}$. Then, $f_{\varphi}^{-1}\left(P_{x}^{v}\right)=P_{w}^{u}$ is a soft point and $f_{\varphi}^{-1}\left(\delta, \mathcal{W}_{2}\right)$ is an infra soft neighborhood of $P_{w}^{u}$. By hypothesis of the infra soft locally connectedness of $\left(\mathcal{U}_{1}, Y_{1}, \mathcal{W}_{1}\right)$, there exists an infra soft connected neighborhood $\left(\lambda, \mathcal{W}_{1}\right) \widetilde{\subseteq} f_{\varphi}^{-1}\left(\delta, \mathcal{W}_{2}\right)$ of $P_{w}^{u}$. It follows from Proposition 8 that $f_{\varphi}\left(\lambda, \mathcal{W}_{1}\right)$ is an infra soft connected neighborhood of $P_{u}^{v}$ such that $f_{\varphi}\left(\lambda, \mathcal{W}_{1}\right) \widetilde{\subseteq}\left(\delta, \mathcal{W}_{2}\right)$. Thus, $\left(\mathcal{U}_{2}, \mathrm{Y}_{2}, \mathcal{W}_{2}\right)$ is infra soft locally connected. Hence, we obtain the desired result.

Theorem 7. The finite product of infra soft locally connected spaces is infra soft locally connected. 
Proof. Let $\left(\mathcal{U}_{1}, Y_{1}, \mathcal{W}_{1}\right)$ and $\left(\mathcal{U}_{2}, Y_{2}, \mathcal{W}_{2}\right)$ be two infra soft locally connected spaces. Let $\left(\xi, \mathcal{W}_{1} \times \mathcal{W}_{2}\right)$ be an infra soft neighborhood of a soft point $P_{(w, x)}^{(u, v)}$ in $\mathcal{U}_{1} \times \mathcal{U}_{2}$. Now, $P_{(w, x)}^{(u, v)}=P_{w}^{u} \times P_{x}^{v}$, and $\left(\xi, \mathcal{W}_{1} \times \mathcal{W}_{2}\right)=\left(\beta, \mathcal{W}_{1}\right) \times\left(\delta, \mathcal{W}_{2}\right)$, where $\left(\beta, \mathcal{W}_{1}\right)$ and $\left(\delta, \mathcal{W}_{2}\right)$ are, respectively, infra soft neighborhoods of soft points $P_{w}^{u} \in \widetilde{\mathcal{U}_{1}}$ and $P_{x}^{v} \in \widetilde{\mathcal{U}_{2}}$. By hypothesis of the infra soft locally connectedness of $\left(\mathcal{U}_{1}, Y_{1}, \mathcal{W}_{1}\right)$ and $\left(\mathcal{U}_{2}, \mathrm{Y}_{2}, \mathcal{W}_{2}\right)$, there exist infra soft connected neighborhoods $\left(\theta, \mathcal{W}_{1}\right)$ and $\left(\lambda, \mathcal{W}_{2}\right)$ of soft points $P_{w}^{u}$ and $P_{x}^{v}$, respectively, such that $\left(\theta, \mathcal{W}_{1}\right) \widetilde{\subseteq}\left(\beta, \mathcal{W}_{1}\right)$ and $\left(\lambda, \mathcal{W}_{2}\right) \widetilde{\subseteq}\left(\delta, \mathcal{W}_{2}\right)$. It follows from Theorem 4 that $\left(\theta, \mathcal{W}_{1}\right) \times\left(\lambda, \mathcal{W}_{2}\right) \widetilde{\subseteq}\left(\beta, \mathcal{W}_{1}\right) \times\left(\delta, \mathcal{W}_{2}\right)=\left(\xi, \mathcal{W}_{1} \times \mathcal{W}_{2}\right)$ is an infra soft connected neighborhood of $P_{(w, x)}^{(u, v)}$ in $\mathcal{U}_{1} \times \mathcal{U}_{2}$. Hence, $\mathcal{U}_{1} \times \mathcal{U}_{2}$ is infra soft locally connected.

\section{Components in the Frame of Infra Soft Topological Spaces}

In this part, we explore the concept of components in the frame of ISTSs and elucidate that the family of all components forms a partition for an ISTS. We show some properties of components in soft topology that are invalid in infra soft topology. Furthermore, we determine the conditions under which the number of components is finite or countable as well as discuss under what conditions the infra soft connected subsets are components. Finally, we investigate the image of the components under infra soft homomorphism maps.

Definition 25. A component of $(\mathcal{U}, Y, \mathcal{W})$ corresponding to $P_{w}^{u}$ is the union of all infra soft connected subsets of $(\theta, \mathcal{W})$ which contain $P_{w}^{u}$. It is denoted by $C\left(P_{w}^{u}\right)$. That is:

$$
C\left(P_{w}^{u}\right)=\widetilde{U}\left\{(\delta, \mathcal{W}) \widetilde{\subseteq} \widetilde{\mathcal{U}}: P_{w}^{u} \in(\delta, \mathcal{W}) \text { and }(\delta, \mathcal{W}) \text { is infra soft connected }\right\} \text {. }
$$

\section{Remark 2.}

(i) According to Corollary 2, every component of a soft point is the largest infra soft connected set containing this soft point;

(ii) If $(\mathcal{U}, Y, \mathcal{W})$ is infra soft connected, then $\tilde{\mathcal{U}}$ is the only component of each soft point.

We present the next example to show the method of calculating a component of each soft point.

Example 7. The family $\mathrm{Y}=\left\{\Phi, \widetilde{\mathcal{U}},\left(\theta_{k}, \mathcal{W}\right): k=1,2,3,4,5\right\}$ is an infra soft topology on $\mathcal{U}=\left\{u_{1}, u_{2}, u_{3}\right\}$ with a set of parameters $\mathcal{W}=\left\{w_{1}, w_{2}\right\}$, where:

$\left(\theta_{1}, \mathcal{W}\right)=\left\{\left(w_{1}, \mathcal{U}\right),\left(w_{2}, \varnothing\right)\right\}$

$\left(\theta_{2}, \mathcal{W}\right)=\left\{\left(w_{1}, \varnothing\right),\left(w_{2}, \mathcal{U}\right)\right\}$

$\left(\theta_{3}, \mathcal{W}\right)=\left\{\left(w_{1}, \varnothing\right),\left(w_{2},\left\{u_{1}\right\}\right)\right\}$

$\left(\theta_{4}, \mathcal{W}\right)=\left\{\left(w_{1},\left\{u_{1}, u_{2}\right\}\right),\left(w_{2}, \varnothing\right)\right\}$, and

$\left(\theta_{5}, \mathcal{W}\right)=\left\{\left(w_{1},\left\{u_{3}\right\}\right),\left(w_{2}, \varnothing\right)\right\}$.

Obviously, $(\mathcal{U}, \mathrm{Y}, \mathcal{W})$ is an infra soft disconnected space. Now, we have the following: $\left(\theta_{2}, \mathcal{W}\right)$.

$C\left(P_{w_{1}}^{u_{1}}\right)=C\left(P_{w_{1}}^{u_{2}}\right)=\left(\theta_{4}, \mathcal{W}\right), C\left(P_{w_{1}}^{u_{3}}\right)=\left(\theta_{5}, \mathcal{W}\right)$ and $C\left(P_{w_{2}}^{u_{1}}\right)=C\left(P_{w_{2}}^{u_{2}}\right)=C\left(P_{w_{2}}^{u_{3}}\right)=$

Theorem 8. The family of all components of any ISTS forms a partition for it.

Proof. Consider $\left\{C\left(P_{w}^{u}\right): P_{w}^{u} \in \widetilde{\mathcal{U}}\right\}$ as a family of all components of an ISTS $(\mathcal{U}, \mathrm{Y}, \mathcal{W})$. It is clear that $\widetilde{\mathcal{U}}=\widetilde{U}\left\{C\left(P_{w}^{u}\right): P_{w}^{u} \in \widetilde{\mathcal{U}}\right\}$. Suppose that there are two distinct soft points $P_{w}^{u}, P_{x}^{v}$ such that $C\left(P_{w}^{u}\right) \widetilde{\cap} C\left(P_{x}^{v}\right) \neq \Phi$. According to Corollary $2,(\theta, \mathcal{W})=C\left(P_{w}^{u}\right) \widetilde{\cup} C\left(P_{x}^{v}\right)$ is an infra soft connected set. This contradicts that $C\left(P_{w}^{u}\right)$ and $C\left(P_{x}^{v}\right)$ are the largest infra soft connected sets containing $P_{w}^{u}$ and $P_{x}^{v}$, respectively. Thus, $C\left(P_{w}^{u}\right) \widetilde{\cap} C\left(P_{x}^{v}\right)=\Phi$. Hence, we obtain the desired result.

The topological result reports that every closed component is one of the results that is invalid in ISTS. 
Proposition 12. For any component $C\left(P_{w}^{u}\right)$ of an $\operatorname{ISTS}(\mathcal{U}, \mathrm{Y}, \mathcal{W})$ we have $C\left(P_{w}^{u}\right)=C l\left(C\left(P_{w}^{u}\right)\right)$.

Proof. Since $C\left(P_{w}^{u}\right)$ is infra soft connected and $C\left(P_{w}^{u}\right) \widetilde{\subseteq} C l\left(C\left(P_{w}^{u}\right)\right)$, it follows from Theorem 2 that $C l\left(C\left(P_{w}^{u}\right)\right)$ is an infra soft connected set as well. However, $C\left(P_{w}^{u}\right)$ is the largest infra soft connected set containing $P_{w}^{u}$; however, $C\left(P_{w}^{u}\right)=C l\left(C\left(P_{w}^{u}\right)\right)$.

In Example 7, the component $C\left(P_{w_{1}}^{u_{1}}\right)=\left(\theta_{4}, \mathcal{W}\right)$ corresponding to $P_{w_{1}}^{u_{1}}$ is an infra soft open set because it is a member of $Y$. However, it is not an infra soft closed set because its complement $\left\{\left(w_{1},\left\{u_{3}\right\}\right),\left(w_{2}, \widetilde{\mathcal{U}}\right)\right\}$ is not infra soft open.

Theorem 9. If the components of an infra soft compact space are infra soft open, then the number of them is finite.

Proof. Let $(\mathcal{U}, \mathrm{Y}, \mathcal{W})$ be infra soft compact. Suppose that the number of components of $(\mathcal{U}, \mathrm{Y}, \mathcal{W})$ is infinite. Now, every component $C_{i}\left(P_{w}^{u}\right)$ is infra soft open and $\left\{C_{i}\left(P_{w}^{u}\right): P_{w}^{u} \in\right.$ $\widetilde{\mathcal{U}}\}$ forms a cover of $\widetilde{\mathcal{U}}$. By hypothesis, $\widetilde{\mathcal{U}}=\widetilde{\cup}_{i=1}^{n}\left\{C_{i}\left(P_{w}^{u}\right): P_{w}^{u} \in \widetilde{\mathcal{U}}\right\}$. This implies that there are some members of the cover that have a non-null intersection; this is a contradiction. Hence, $(\mathcal{U}, \mathrm{Y}, \mathcal{W})$ has a finite number of components.

Corollary 4. If the components of an infra soft Lindelöf space are infra soft open, then the number of them is countable.

Theorem 10. If an ISTS has a finite number of components which are infra soft closed, then they are also infra soft open.

Proof. Consider $(\mathcal{U}, \mathrm{Y}, \mathcal{W})$ as an ISTS with a finite number of components which are infra soft closed. As we previously proved that the family of components forms a partition for $\widetilde{\mathcal{U}}$. Since every component is infra soft closed, the union of any number of them is infra soft closed. Since $\widetilde{U}_{i=2}^{n} C_{i}\left(P_{w}^{u}\right)$ is infra soft closed, then $C_{1}\left(P_{w}^{u}\right)$ is infra soft open. This completes the proof.

Theorem 11. If $(\theta, \mathcal{W})$ is a non-null infra soft clopen and infra soft connected subset of $(\mathcal{U}, Y, \mathcal{W})$, then $(\theta, \mathcal{W})$ is a component.

Proof. Let the given condition be satisfied. Suppose that $(\delta, \mathcal{W})$ is infra soft connected such that $(\theta, \mathcal{W}) \widetilde{\subseteq}(\delta, \mathcal{W})$. Now, $(\delta, \mathcal{W})=(\theta, \mathcal{W}) \widetilde{\cup}\left[\left(\theta^{c}, \mathcal{W}\right) \widetilde{\bigcap}(\delta, \mathcal{W})\right]$. Since $(\theta, \mathcal{W})$ is a non-null infra soft clopen set, $C l(\theta, \mathcal{W}) \widetilde{\bigcap}\left[\left(\theta^{c}, \mathcal{W}\right) \widetilde{\bigcap}(\delta, \mathcal{W})\right]=\Phi$ and $(\theta, \mathcal{W}) \widetilde{\bigcap} C l\left[\left(\theta^{c}, \mathcal{W}\right) \widetilde{\bigcap}(\delta, \mathcal{W})\right]=$ $\Phi$. Therefore, $(\theta, \mathcal{W})$ and $\left(\theta^{c}, \mathcal{W}\right) \widetilde{\cap}(\delta, \mathcal{W})$ are disconnection soft sets of $(\delta, \mathcal{W})$. This contradicts the assumption. Hence, $(\theta, \mathcal{W})$ is a component.

Proposition 13. The infra soft homeomorphism image of a component is also a component.

Proof. Consider $f_{\varphi}:\left(\mathcal{U}_{1}, \mathrm{Y}_{1}, \mathcal{W}_{1}\right) \rightarrow\left(\mathcal{U}_{2}, \mathrm{Y}_{2}, \mathcal{W}_{2}\right)$ as an infra soft homeomorphism map and let $\left(\theta, \mathcal{W}_{1}\right)$ be a component of $\left(\mathcal{U}_{1}, \mathrm{Y}_{1}, \mathcal{W}_{1}\right)$. Suppose that $f_{\varphi}\left(\theta, \mathcal{W}_{1}\right)$ is not a component of $\left(\mathcal{U}_{2}, Y_{2}, \mathcal{W}_{2}\right)$. This means, according to Proposition 8 , that there is an infra soft connected subset $\left(\delta, \mathcal{W}_{2}\right)$ of $\left(\mathcal{U}_{2}, Y_{2}, \mathcal{W}_{2}\right)$ such that $f_{\varphi}\left(\theta, \mathcal{W}_{1}\right) \widetilde{\subseteq}\left(\delta, \mathcal{W}_{2}\right)$. Since $f_{\varphi}$ is an infra soft homeomorphism, $\left(\theta, \mathcal{W}_{1}\right) \widetilde{\widetilde{c}} f_{\varphi}^{-1}\left(\delta, \mathcal{W}_{2}\right)$. Now, $f_{\varphi}^{-1}\left(\delta, \mathcal{W}_{2}\right)$ is an infra soft connected subset of $\left(\mathcal{U}_{1}, \mathrm{Y}_{1}, \mathcal{W}_{1}\right)$ which contradicts that $\left(\theta, \mathcal{W}_{1}\right)$ is a component. Hence, $f_{\varphi}\left(\theta, \mathcal{W}_{1}\right)$ is a component.

\section{Concluding Remark and Further Work}

In this work, we continue displaying and studying the essential ideas in the frame of ISTSs. The main goal of this article was to define the concepts of infra soft connected and infra soft locally connected spaces and components. We characterized them and showed that the concepts of infra soft connected and infra soft locally connected spaces are 
independent of each other; we also determined the conditions under which the infra soft connected subsets are components.

According the obtained results, many classical properties of these concepts are still valid for infra soft topological structures. The benefit of this matter is to study and reveal the interrelationships among these concepts in the frame of ISTSs instead of soft topologies which means cancel unnecessary conditions of a soft topology.

On the other hand, we showed that a few properties of infra soft locally connected spaces and components in soft topology are invalid in infra soft topology. For example, (1) every component is infra soft closed; and (2) if an ISTS has a finite number of components, then these components are infra soft clopen sets. Furthermore, we provide some examples to confirm that there is no relationship between infra soft topology and its parametric infra topologies with respect to possessing the property of infra soft connectedness.

As a future work, we can intensively study the concepts of infra soft locally connected spaces and components with respect to the ordinary points instead of soft points. In fact, this new path will lead to initiate different types of these concepts due to the kinds of belonging relations: partial or total. However, we anticipate that some properties of these concepts will be lost in ISTSs. Furthermore, we are going to study different types of infra soft connectedness such as infra soft hyperconnectedness, infra soft ultraconnectedness and extremely infra soft disconnectedness.

Author Contributions: The two authors have the same contributions. Both authors have read and agreed to the published version of the manuscript.

Funding: This research received no external funding.

Institutional Review Board Statement: Not applicable.

Informed Consent Statement: Not applicable.

Data Availability Statement: Not applicable.

Conflicts of Interest: The authors declare no conflict of interest.

\section{References}

1. Molodtsov, D. Soft set theory-First results. Comput. Math. Appl. 1999, 37, 19-31. [CrossRef]

2. Yang, J.; Yao, Y. Semantics of soft sets and three-way decision with soft sets. Knowl. Based Syst. 2020, 194, 105538. [CrossRef]

3. El-Bably, M.K.; Atik, A.A.E. Soft $\beta$-rough sets and its application to determine COVID-19. Turk. J. Math. 2021, 45, 1133-1148. [CrossRef]

4. Kharal, A.; Ahmed, B. Mappings on soft classes. New Math. Nat. Comput. 2011, 7, 471-481. [CrossRef]

5. Korei, A.; Radeleczki, S.; Szilagyi, S. An accuracy-type order on rough sets. Miskolc Math. Notes 2019, 20, 331-343. [CrossRef]

6. Xian, Z.; Chen, J.; Yu, P. Relative relation matrix-based approaches for updating approximations in multigranulation rough sets. Filomat 2020, 34, 2253-2272. [CrossRef]

7. Çağman, N.; Enginoxgxlu, S. Soft matrix theory and its decision making. Comput. Math. Appl. 2010, 59, 3308-3314. [CrossRef]

8. Maji, P.K.; Biswas, R.; Roy, R. Soft set theory. Comput. Math. Appl. 2003, 45, 555-562. [CrossRef]

9. Ali, M.I.; Feng, F.; Liu, X.; Min, W.K.; Shabir, M. On some new operations in soft set theory. Comput. Math. Appl. 2009, 57, 1547-1553. [CrossRef]

10. Al-shami, T.M.; El-Shafei, M.E. T-soft equality relation. Turk. J. Math. 2020, 44, 1427-1441. [CrossRef]

11. Qin, K.; Hong, Z. On soft equality. J. Comput. Appl. Math. 2010, 234, 1347-1355. [CrossRef]

12. Çă̆man, N.; Karataş, S.; Enginoglu, S. Soft topology. Comput. Math. Appl. 2011, 62, 351-358. [CrossRef]

13. Shabir, M.; Naz, M. On soft topological spaces. Comput. Math. Appl. 2011, 61, 1786-1799. [CrossRef]

14. Al-shami, T.M.; Kočinac, L.D.R. The equivalence between the enriched and extended soft topologies. Appl. Comput. Math. 2019, $18,149-162$.

15. Alcantud, J.C.R. Soft open bases and a novel construction of soft topologies from bases for topologies. Mathematics $2020,8,672$. [CrossRef]

16. Al-Ghour, S. Soft $\omega^{*}$-paracompactness in soft topological spaces. Int. J. Fuzzy Log. Intell. Syst. 2021, 21, 57-65. [CrossRef]

17. El-Sheikh, S.A.; El-Latif, A.M.A. Decompositions of some types of supra soft sets and soft continuity. Int. J. Math. Trends Technol. 2014, 9, 37-56. [CrossRef]

18. Ittanagi, B.M. Soft bitopological spaces. Int. J. Comput. Appl. 2014, 107, 1-4.

19. Al-shami, T.M. New soft structure: Infra soft topological spaces. Math. Probl. Eng. 2021, 2021. [CrossRef]

20. Hussain, S. A note on soft connectedness. J. Egypt. Math. Soc. 2015, 23, 6-11. [CrossRef] 
21. Hussain, S. Binary soft connected spaces and an application of binary soft sets in decision making problem. Fuzzy Inf. Eng. 2019, 11, 506-521. [CrossRef]

22. Lin, F. Soft connected spaces and soft paracompact spaces. Int. J. Math. Sci. Eng. 2013, 7, 1-7.

23. Das, S.; Samanta, S.K. Soft metric. Ann. Fuzzy Math. Inform. 2013, 6, 77-94.

24. El-Shafei, M.E.; Abo-Elhamayel, M.; Al-shami, T.M. Partial soft separation axioms and soft compact spaces. Filomat 2018, 32, 4755-4771. [CrossRef]

25. Nazmul, S.; Samanta, S.K. Neighbourhood properties of soft topological spaces. Ann. Fuzzy Math. Inform. 2013, 6, 1-15.

26. Aygünoğlu, A.; Aygxuxn, H. Some notes on soft topological spaces. Neural Comput. Appl. 2012, 21, 113-119. [CrossRef]

27. Feng, F.; Li, Y.M.; Davvaz, B.; Ali, M.I. Soft sets combined with fuzzy sets and rough sets: A tentative approach. Soft Comput. 2010, 14, 899-911. [CrossRef]

28. Al-shami, T.M. Infra soft compact spaces and application to fixed point theorem. J. Funct. Spaces 2021, $2021,3417096$. 